\title{
Forma de acceso a los Servicios de Urgencia hospitalarios y pertinencia de la consulta
}

\author{
Santiago Reinoso Hermidaa, Gabriel J. Díaz Grávalos ${ }^{b}$, Aida Robles Castiñeirasc, \\ Elena Pereiro Sánchez ${ }^{d}$, Eduardo Fernández Cardamae, Ana López González ${ }^{\dagger}$
}

a Médico de Familia, PAC O Carballiño (Ourense).

${ }^{\mathrm{b}}$ Médico de Familia, C.S. Cea (Ourense).

${ }^{\mathrm{c}}$ Médico de Familia, PAC Xinzo de Limia (Ourense).

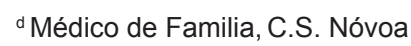
Santos-Ourense (Ourense).

${ }^{e}$ Médico de Familia, PAC Lalín (Pontevedra).

${ }^{\mathrm{f}}$ MIR Medicina de Familia y Comunitaria, C.S.

Mariñamansa-Ourense (Ourense).

Correspondencia: Santiago

Reinoso Hermida.

Correo electrónico:

Santiago.reinoso.hermida@sergas.es.

Recibido el 23 de junio de 2011.

Aceptado para su publicación el 29 de agosto de 2011.

\section{RESUMEN}

Objetivo. Conocer las diferentes formas de acceso a los Servicios de Urgencia Hospitalarios (SUH), valorar la adecuación de las consultas allí atendidas y analizar la posible asociación entre forma de acceso y adecuación, así como con otros factores relacionados.

Diseño. Estudio observacional transversal.

Emplazamiento. Servicio de urgencias hospitalario.

Participantes. Pacientes que acuden al SUH, espontáneamente o derivados por Servicios de Urgencia Extrahospitalarios (SUE) o médico de atención primaria (MAP). No hubo negativas a contestar.

Mediciones principales. Cuestionario elaborado ad hoc con 14 variables: sociodemográficas y otras relacionadas con la forma de remisión y el tipo de consulta en el SUH. La encuesta fue llevada a cabo en el SUH por los investigadores que prestaban la asistencia a la totalidad de los pacientes atendidos por ellos en cada jornada de servicio.

Resultados. Fueron analizadas 264 encuestas. El 77,6\% (205) de los casos se corresponde con cuadros agudos de inicio y patología de tipo traumatológico. En 74 casos $(28,0 \%)$ el paciente había consultado previamente a su MAP por este mismo motivo. En los pacientes remitidos por su MAP, en el $68,4 \%$ de los casos procedía la consulta en el SUH. En los pacientes que acudían espontáneamente este porcentaje era del $25,7 \%$. No se constataron diferencias en el porcentaje de pacientes en los que no procedía consulta urgente. Los pacientes que acudían espontáneamente al SUH eran más jóvenes (diferencia de medias 9,2 años; IC 95\%: 3,4 - 14,9; $p=0,001)$ y procedían mayoritariamente del medio urbano $\left(x^{2}=9,8 ; p=0,002\right)$.

Conclusiones. La mayoría de los pacientes que demandan atención urgente lo hacen mediante el bypass de los SUE, fenómeno influido por la procedencia urbana, una menor edad y la existencia de ingresos previos. La remisión por SUE o MAP mejora la pertinencia de la consulta en los SUH.

Palabras Clave. Actitud frente a la Salud, Servicios Médicos de Urgencia, Salud Rural.

\section{ABSTRACT}

Means of access to hospital emergency departments and relevance of the consultation Objective. To determine the different means of access to hospital emergency departments (ED), assess the appropriateness of consultations given there and analyze the possible association between access and appropriateness as well as other related factors.

Design. Observational cross-sectional study.

Setting. Hospital Emergency Departments.

Participants. Patients who go to the ED on their own accord or are referred by Prehospital Emergency Care (PEC) services or a General Practitioner (GP). No-one refused to answer the questionnaire.

Measurements. Ad hoc questionnaire with 14 variables: socio-demographic and others related with the means of access and type of consultation in the ED. The survey was conducted in the ED by the researchers who provided care to all patients seen by them on each shift.

Results. A total of 264 surveys were analyzed. $77.6 \%$ (205) of cases were due to acute episodes of disease onset and trauma related events. At total of $74(28.0 \%)$ patients had previously consulted their GP for the same reason. For $68.4 \%$ of the patients referred by their GP and $25 \%$ of the patients who came on their own accord the consultation in the ED was appropriate. There were no differences in the percentage of patients whose emergency consultation was not appropriate. Patients who came to ED on their own accord were younger (mean difference 9.2 years, $95 \% \mathrm{Cl}: 3.4-14.9, p=0.001)$ and mostly came from urban areas $(x 2=9.8, p=0.002)$.

Conclusions. Most patients requiring emergency care access the ED through the PEC service, this is influenced by the urban origin, younger age and the existence of previous admissions. Referrals by PEC or GP improves the appropriateness of the consultations in the ED.

Key Words. Attitude to Health. Emergency Medical Services, Rural Health.

Un avance de este estudio se presentó como Comunicación Oral en las XIV Jornadas Gallegas de Medicina de Familia y Comunitaria (Ourense, mayo de 2009). 


\section{INTRODUCCIÓN}

Del mismo modo que en el Sistema Nacional de Salud la Atención Primaria es la puerta de entrada al sistema, cabría esperar que en la atención urgente el acceso se efectuara a través de su equivalente, los Servicios de Urgencias Extrahospitalarios (SUE). Sin embargo, un alto porcentaje de pacientes acuden directamente a los Servicios de Urgencia Hospitalarios (SUH), en una práctica conocida internacionalmente como bypass, demandando atención en el segundo nivel por patologías que en muchas ocasiones no lo necesitan, lo que contribuye a la saturación de los distintos servicios, el descenso de la calidad asistencial ${ }^{1} \mathrm{y}$ al aumento del gasto sanitario².

Entre 1997 y 2005, en España, se ha constatado un aumento de la asistencia a los SUH de un 2,6\% anual, lo que en cifras absolutas supone 720.000 demandas adicionales de asistencia al año ${ }^{3}$. Este incremento es debido en su mayor parte a pacientes que utilizan de manera inadecuada los $\mathrm{SUH}^{4}$, hipótesis que se ve reforzada por la baja tasa de ingresos en relación con el número de consultas.

La frecuentación a los SUH es muy variable en función de las distintas Comunidades Autónomas e incluso, dentro de éstas, de sus diferentes Áreas de Salud, lo que hace pensar que los habitantes hacen un uso diferencial de los SUH en función de patrones locales y no de aspectos puramente médicos ${ }^{5}$.
Diversos estudios han caracterizado a esos pacientes, describiendo los factores sociodemográficos, el tipo de patología e incluso los motivos esgrimidos para acudir a los SUH como primera opción, así como la pertinencia o no de la consulta.

Los objetivos del estudio fueron conocer las diferentes formas de acceso a un SUH, valorar la adecuación de las consultas atendidas en un SUH y analizar la posible asociación entre la forma de acceso y la adecuación de las consultas atendidas en un SUH, así como con otros factores relacionados.

\section{MATERIAL Y MÉTODO}

Estudio observacional transversal. Se realizó una encuesta a pacientes que acuden al SUH, espontáneamente o derivados por SUE o médico de atención primaria (MAP). El citado SUH pertenece a un centro de $2^{\circ}$ nivel, de ámbito provincial, en el que las urgencias obstétricas y pediátricas son atendidas por servicios de urgencias específicos. La demanda asistencial fue de 302 urgencias al día en 2008. La encuesta fue llevada a cabo en el SUH por los investigadores que prestaban la asistencia a la totalidad de los pacientes atendidos por ellos en cada jornada de servicio. Se explicó la finalidad de la encuesta a los pacientes y se solicitó su participación. No hubo negativas globales a contestar.

\begin{tabular}{|c|c|}
\hline Variables & Categorías \\
\hline $\begin{array}{ll}\text { Variables cualitativas } \\
- & \text { Sexo } \\
- & \text { Nivel educativo } \\
- & \text { Estado civil } \\
- & \text { Medio de procedencia } \\
- & \text { Disponibilidad de vehículo } \\
- & \text { Ingresos previos } \\
- & \text { Pertinencia de la consulta en el SUH según } \\
\text { - } & \text { l facultativo que presta la asistencia } \\
& \text { Motivos alegados por el paciente para } \\
- & \text { Forma de acceso al SUH } \\
- & \text { Motivo de la consulta en el SUH } \\
- & \text { Consulta previa al MAP por ese motivo } \\
- & \text { Diagnóstico al alta }\end{array}$ & $\begin{array}{c}\text { Hombre / mujer } \\
\text { Estudios primarios / secundarios / universitarios } \\
\text { Soltero o separado / casado / viudo } \\
\text { Rural / urbano } \\
\text { Sí / no } \\
\text { Sí / no } \\
\text { No precisa consulta urgente / procede consulta en SUE / procede } \\
\text { consulta en SUH } \\
\text { Remisión por el sistema (MAP, SUE) / más medios, más confianza / } \\
\text { enfermo crónico o gravedad percibida / comodidad, cercanía, hábito } \\
\text { Remitido (MAP, SUE) / espontáneamente } \\
\text { Abierta } \\
\text { Sí / no } \\
\text { Abierta }\end{array}$ \\
\hline $\begin{array}{l}\text { Variables cuantitativas } \\
-\quad \text { Edad } \\
-\quad \text { Tiempo de viaje hasta el hospital }\end{array}$ & $\begin{array}{l}\text { Años cumplidos } \\
\text { En minutos }\end{array}$ \\
\hline
\end{tabular}

Tabla 1. Variables recogidas en el cuestionario y categorías de respuesta. 


\begin{tabular}{|c|c|c|c|}
\hline Variable & $\begin{array}{c}\text { Varón } \\
137(51,9 \%)\end{array}$ & $\begin{array}{c}\text { Mujer } \\
127(48,1 \%)\end{array}$ & $\begin{array}{l}\text { Total } \\
264\end{array}$ \\
\hline Edad [años; (DE)] & $50,5(21,3)$ & $53,0(23,6)$ & $51,7(22,4)$ \\
\hline $\begin{array}{ll}\text { Estado civil } \\
- & \text { Soltero o separado } \\
- & \text { Casado } \\
- & \text { Viudo }\end{array}$ & $\begin{array}{c}46(33,6 \%) \\
83(60,6 \%) \\
8(5,8 \%)\end{array}$ & $\begin{array}{l}40(31,5 \%) \\
70(55,1 \%) \\
17(13,4 \%)\end{array}$ & $\begin{array}{c}86(32,6 \%) \\
153(58,0 \%) \\
25(9,4 \%)\end{array}$ \\
\hline $\begin{array}{ll}\text { Nivel educativo } \\
\text { - } & \text { Estudios primarios } \\
\text { - } & \text { Estudios secundarios } \\
\text { - } & \text { Estudios universitarios }\end{array}$ & $\begin{array}{c}76(55,5 \%) \\
48(35,0 \%) \\
13(9,5 \%)\end{array}$ & $\begin{array}{l}83(65,4 \%) \\
31(24,4 \%) \\
13(10,2 \%)\end{array}$ & $\begin{array}{c}159(60,2 \%) \\
79(29,9 \%) \\
26(9,8 \%)\end{array}$ \\
\hline $\begin{array}{ll}\text { Medio de procedencia } \\
-\quad \text { Rural } \\
-\quad \text { Urbano }\end{array}$ & $\begin{array}{l}70(51,1 \%) \\
67(48,9 \%)\end{array}$ & $\begin{array}{l}63(49,6 \%) \\
64(50,4 \%)\end{array}$ & $\begin{array}{l}133(50,4 \%) \\
131(49,6 \%)\end{array}$ \\
\hline $\begin{array}{l}\text { Disponibilidad de vehículo } \\
-\quad \text { Sí } \\
-\quad \text { No }\end{array}$ & $\begin{array}{c}116(84,7 \%) \\
21(15,3 \%)\end{array}$ & $\begin{array}{c}106(83,5 \%) \\
21(16,5 \%)\end{array}$ & $\begin{array}{c}222(84,1 \%) \\
40(15,9 \%)\end{array}$ \\
\hline $\begin{array}{l}\text { Ingresos previos } \\
-\quad \text { Sí } \\
-\quad \text { No }\end{array}$ & $\begin{array}{l}70(51,1 \%) \\
67(48,9 \%)\end{array}$ & $\begin{array}{l}94(74,0 \%) \\
33(26,1 \%)\end{array}$ & $\begin{array}{l}164(62,1 \%) \\
100(37,9 \%)\end{array}$ \\
\hline Tiempo de viaje hasta el hospital [minutos; (DE)] & $18,1(13,7)$ & $18,2(13,1)$ & $18,2(13,4)$ \\
\hline
\end{tabular}

Tabla 2. Variables sociodemográficas de los pacientes incluidos.

\begin{tabular}{l|c}
\hline Variable & $\mathbf{N}^{\circ}(\%)$ \\
\hline Pertinencia de la consulta en el SUH según el facultativo que presta la asistencia & \\
- No precisa consulta urgente & $41(15,5 \%)$ \\
- Procede consulta en SUE & $139(52,7 \%)$ \\
- Procede consulta en SUH & $84(31,8 \%)$ \\
Motivos alegados por el paciente para acudir al SUH* & \\
- Remisión por el Sistema (MAP, SUE) & $93(35,6 \%)$ \\
- Más medios/más confianza & $93(35,6 \%)$ \\
- Enfermo crónico o gravedad percibida & $18(6,9 \%)$ \\
\hline
\end{tabular}

Tabla 3. Datos relacionados con la presencia del paciente en el Servicio de Urgencias Hospitalario. * Tres pacientes no contestaron esta pregunta. SUE: servicio de urgencias extrahospitalario; SUH: servicio de urgencias hospitalario; MAP: médico de atención primaria.

Se administró un cuestionario elaborado ad hoc constituido por 14 preguntas que definían 14 variables: sociodemográficas y otras relacionadas con la forma de remisión y el tipo de consulta en el SUH. La variable forma de acceso al SUH se clasificó en 3 categorías: según los pacientes acudieran espontáneamente o fueran derivados por su MAP o un SUE (tabla 1). Se hizo una prueba piloto en 10 pacientes para valorar la factibilidad del protocolo, el tiempo requerido por paciente y corregir posibles errores de la hoja de recogida de datos de las variables relacionadas.

El análisis bivariante se realizó mediante comparación de proporciones y medias $\left(X^{2}\right.$ y t-Student). Se realizó un análisis multivariante mediante regresión logística. La variable forma de acceso (espontánea o derivado) se consideró variable dependiente. Fueron incluidas como variables independientes edad, sexo, procedencia 


\begin{tabular}{|c|c|c|c|c|}
\hline \multirow[t]{2}{*}{ Variable } & \multicolumn{2}{|c|}{ Forma de acceso al SUH } & \multirow[t]{2}{*}{$\mathbf{X}^{2}$} & \multirow[t]{2}{*}{$\mathbf{p}$} \\
\hline & Remitido* & Espontáneamente & & \\
\hline \multicolumn{5}{|l|}{ Edad } \\
\hline$-\quad<65$ años & $51(29,8 \%)$ & $120(70,2 \%)$ & 6,2 & 0,01 \\
\hline - $\quad \geq 65$ años & $42(45,2 \%)$ & $51(54,8 \%)$ & & \\
\hline \multicolumn{5}{|l|}{ Sexo } \\
\hline - Varón & $54(39,4 \%)$ & $83(60,6 \%)$ & 2,2 & 0,14 \\
\hline - $\quad$ Mujer & $39(30,7 \%)$ & $88(69,3 \%)$ & & \\
\hline \multicolumn{5}{|l|}{ Pertinencia consulta } \\
\hline - $\quad$ No precisa & $8(19,5 \%)$ & $33(80,5 \%)$ & 42,1 & $<0,001$ \\
\hline - $\quad$ Precisa SUE & $32(23,0 \%)$ & $107(77,0 \%)$ & & \\
\hline - $\quad$ Precisa SUH & $53(63,1 \%)$ & $31(36,9 \%)$ & & \\
\hline
\end{tabular}

Tabla 4. Forma de acceso al Servicio de Urgencias Hospitalario en función de diferentes variables consideradas. *Remitido por Médico de Atención Primaria, SUE o sistema de emergencias (061); SUH: Servicio de Urgencias hospitalario; SUE: Servicio de Urgencias extrahospitalario.

\begin{tabular}{l|ccc}
\hline Variable & OR & IC95 & p \\
\hline Edad & 0,98 & $0,97-0,99$ & 0,001 \\
Procedencia* $_{\text {Ingresos previos** }}$ & 2,2 & $1,3-3,9$ & 0,005 \\
& 2,3 & $1,2-4,2$ & 0,025 \\
\hline
\end{tabular}

Tabla 5. Resultados de la regresión logística considerando como variable dependiente la remisión al SUH. Categorías de referencia: * Rural; ${ }^{* *}$ No.

(rural o urbana), existencia de ingresos previos, nivel educativo y estado civil. Se consideraron significativos valores de $p<0,05$.

\section{RESULTADOS}

Fueron analizadas 264 encuestas. La tabla 2 refleja las características sociodemográficas de los pacientes encuestados. En la tabla 3 figuran los datos relacionados con la presencia del paciente en el SUH.

La proporción de pacientes que acudieron espontáneamente al SUH, sin ser remitidos por su MAP o SUE, fue del 64,8\% (IC 95\%: 58,8-70,7). Los pacientes que acudían espontáneamente al SUH eran más jóvenes (diferencia de medias 9,2 años; IC 95\%: 3,4 - 14,9; p = 0,001) y procedían mayoritariamente del medio urbano $(55,6 \%$ frente a 74,0\%; $\left.x^{2}=9,8 ; p=0,002\right)$. No se encontraron diferencias en la forma de acceso por sexo $(60,6 \%$ en varones frente a $69,3 \%$ en mujeres; $x^{2}=2,2 ; p$ $=0,14)$.
En función de la edad (mayores y menores de 65 años) no se observan diferencias en la pertinencia de la consulta en el grupo de pacientes que no precisaban consulta urgente según los médicos que prestaban asistencia. Sí que se constatan entre aquellos en los que procedía algún tipo de consulta urgente, siendo más frecuente la pertinencia de consulta hospitalaria en los mayores de 65 años $\left(46,2 \%\right.$ frente a $\left.24,0 \% ; x^{2}=14,1 ; p=0,001\right)$.

En los pacientes remitidos por su MAP, en el $68,4 \%$ de los casos procedía la consulta en el SUH. En el caso de los pacientes que acudían espontáneamente este porcentaje era del $25,7 \%$. No se constataron diferencias en el porcentaje de pacientes en los que no procedía consulta urgente independientemente de que fueran derivados por sus MAP o acudieran espontáneamente (15,8\% frente a $15,5 \%$ ).

La tabla 4 muestra la forma de acceso al SUH en función de diferentes variables consideradas y la tabla 5 muestra el resultado de la regresión logística, considerando como variable dependiente 
la remisión o no por el sistema sanitario. El análisis de los diagnósticos realizados en el momento del alta del paciente muestra que el 77,6\% (205) de los casos se corresponde con cuadros agudos de inicio y patología de tipo traumatológico. En 74 casos $(28,0 \%)$ el paciente había consultado previamente a su médico MAP por este mismo motivo.

\section{DISCUSIÓN}

En este estudio, dos tercios de los pacientes que acudieron al SUH lo hicieron sin consultar previamente a su MAP ni a un SUE y sólo en la tercera parte de los pacientes el facultativo del SUH considera adecuada la consulta a este servicio, pertinencia que se duplica en el grupo de los que han sido derivados por su MAP o un SUE. Este dato es similar a los obtenidos por Torné et al. ${ }^{6}$, y difiere de los encontrados en la mayoría de estudios que utilizaron el Protocolo de Adecuación de Urgencias Sanitarias (PAUH) como sistema de valoración de la pertinencia de la consulta ${ }^{7,8}$.

Los pacientes más jóvenes son los que más acuden de forma espontánea a los SUH, dato similar a otras series $^{4,8,9}$, en las que además se aprecia cómo ese uso es inadecuado en su mayor parte. Es posible que los pacientes ancianos utilicen más los SUE y los MAP en base a una mayor confianza en su médico de cabecera y a una menor capacidad para desplazarse ${ }^{10,11}$. Además, su uso es más apropiado al padecer patologías más graves que requieren asistencia urgente en la reagudización de los procesos crónicos ${ }^{12}$.

Los dos argumentos más utilizados para el acceso espontáneo a los SUH son la mayor confianza y disponibilidad de medios en los hospitales y la comodidad de acceso o costumbre, motivos similares a los argumentados en otros trabajos ${ }^{4,13}$. La circunstancia de que los pacientes residentes en medio urbano sean los que más acuden de forma espontánea a los SUH parece guardar relación con la comodidad esgrimida como argumento. El hecho de que en el medio rural los SUE compartan habitualmente ubicación con los centros de Atención Primaria (AP) puede servir de referencia física para la asistencia sanitaria urgente en esas zonas, mientras que en el ámbito de nuestro estudio el SUE urbano no se corresponde con ninguno de los centros de AP de la ciudad, lo que puede que esté generando un desconocimiento de esa oferta de servicios. Podría parecer lógico que el tiempo de desplazamiento desde el lugar de residencia hasta el SUH influyera en la mayor demanda de los pacientes del medio urbano, pero en este caso no se ha encontrado esta asociación.

Algunos autores vinculan el bypass de los SUE con la dificultad para acceder a estos servicios, bien sea a la hora de conseguir cita previa o por el horario de atención ${ }^{9}$, o por la existencia de barreras de acceso a otros servicios ${ }^{14}$. No obstante, esto no es aplicable en nuestro entorno, ya que en todos los centros se atiende de forma urgente, aunque esa demanda sea inapropiada. El tiempo medio para la cita en medicina general hasta el tercer hueco libre en la agenda del día (calculado a las 8:00 h a.m.) es de 0,57 días $^{15}$.

Por otro lado, se pudo comprobar la asociación entre la existencia de ingresos previos y el acceso de forma espontánea, como también ha sido constatado en otras series ${ }^{11}$. Este fenómeno puede ser explicado por la impresión subjetiva del paciente de que será mejor atendido en el centro hospitalario en el que ya ha estado ingresado, generando una sensación de confianza.

Según los resultados obtenidos, si todos los pacientes acudieran en primera instancia a los SUE como filtro ante el SUH, la demanda en este último bajaría en torno a una tercera parte, aun incluyendo a aquellos pacientes derivados improcedentemente desde AP. Sin embargo, autores como Oterino de la Fuente et $\mathrm{al}^{16}$. sugieren que un aumento de los SUE no reduce la frecuentación a los SUH. Curiosamente el porcentaje de pacientes en los que no procedía ningún tipo de asistencia urgente era el mismo independientemente de la forma de acceso. En el trabajo de Sempere et al. ${ }^{4}$ se aprecian cifras de derivación incorrecta similares a las obtenidas en nuestra muestra. No obstante, es importante hacer hincapié en que, en la mayoría de pacientes del grupo que acudió espontáneamente, la consulta no procedía en SUH sino en SUE, mientras que en los derivados desde AP si procedía dicha consulta, lo cual es resaltado en otros trabajos ${ }^{7}$.

Un medio para conseguir evitar el bypass y mejorar la pertinencia de las consultas en los SUH podría ser el experimentado por Miró et al $^{17}$, en el que se demuestra cómo es posible derivar a un centro externo a un importante número de consultas menores sin visita previa, con un adecuado triage, sin que ello suponga un riesgo para los pacientes.

Los resultados deben ser considerados teniendo en cuenta las limitaciones inherentes a este tipo de 
encuestas. No se comprobó que las respuestas de los pacientes se correspondieran con la realidad, existiendo la posibilidad de que el paciente, como en toda encuesta, falseara la realidad, lo que es un problema propio de los estudios realizados mediante este sistema. La selección no aleatoria de los facultativos puede sesgar los resultados, del mismo modo que el hecho de que no todos los facultativos del turno participasen en el estudio, sin embargo, la asignación de cada paciente en el SUH al facultativo se realiza por una tercera persona, lo que aleatoriza la muestra.

En el presente estudio la pertinencia fue valorada de modo subjetivo por los facultativos que prestaban asistencia en el SUH. En los otros estudios reseñados anteriormente se han utilizado herramientas como el $\mathrm{PAUH}^{18}$, que, pese a parecer un medio más objetivo, sobrevalora la pertinencia de las visitas debido a la práctica habitual en los SUH de la solicitud de pruebas diagnósticas en paralelo, en lugar de en serie en función de resultados previos ${ }^{10}$. Además, el PAUH presenta una baja validez al comparar los resultados con las opiniones de un grupo de expertos, que consideran como inapropiadas un mayor número de visitas ${ }^{19}$.

En conclusión, la mayoría de los pacientes que demandan atención urgente lo hacen mediante el bypass de los SUE, fenómeno influido por la procedencia urbana, una menor edad y la existencia de ingresos previos. La remisión por SUE o MAP mejora la pertinencia de la consulta en los SUH. A la vista de los resultados, sería interesante plantear medidas de actuación a aplicar sobre las variables relacionadas con el bypass de los SUE y, asimismo, estudiar el efecto que producen a medio y largo plazo.

\section{BIBLIOGRAFÍA}

1. Miró $O$, Antonio $M T$, Jiménez $S$, De Dios $A$, Sánchez $M$, Borrás $A$, et al. Decreased health care quality associated with emergency department overcrowding. Eur J Emerg Med. 1999; 6:105-7.

2. Williams RM. The cost of visits to emergency departments. N Engl J Med. 1996; 334:642-6.

3. Rivas Ruiz F, Perea-Milla E, Jiménez-Puente A, Rodríguez del Águila MM, Librero López J, Rebollo García N, et al. Evolución de la utilización de los servicios de urgencias hospitalarios en España en el periodo 1997-2005. Gac Sanit. 2008; 22(Espec Congr):47-8.
4. Sempere Selva T, Peiró S, Sendra Pina P, Martínez Espín C, López Aguilera I. Inappropriate use of an accident and emergency department: magnitude, associated factors and reasons-an approach with explicit criteria. Ann Emerg Med. 2001; 37:568-79.

5. Peiró S, Librero J, Ridao M, Bernal-Delgado E. Grupo de Variaciones en la Práctica Médica en el Sistema Nacional de Salud. Variabilidad en la utilización de los servicios de urgencias hospitalarios del Sistema Nacional de Salud. Gac Sanit. 2010; 24:6-12.

6. Torné E, Guarga A, Torras MG, Pozuelo A, Pasarin M, Borrell C. Análisis de la demanda en servicios de urgencias de Barcelona. Aten Primaria. 2003; 32:423-9.

7. Ochoa FJ, Ramalle-Gomara E, Villar A, Ruiz J, Bragado C, Gimeno C. Visitas inapropiadas al servicio de urgencias de un hospital general. Med Clin (Barc). 2000; 115:377-8.

8. Oterino de la Fuente D, Peiró S, Calvo Rico R, Sutil P, Fernández $O$, Pérez $G$ et al. Utilización inadecuada de un servicio de urgencias hospitalario. Una evaluación con criterios explícitos. Gac Sanit. 1999; 13:361-70.

9. Carret ML, Fassa AG, Kawachi I. Demand for emergency health service: factors associated with inapropiate use. BMC Health Serv Res. 2007; 7:131.

10. Aranaz JM, Martínez R, Rodrigo V, Gómez F, Antón P. Adecuación de la demanda de atención sanitaria en servicios de urgencias hospitalarios. Med Clin (Barc). 2004; 123:615-8.

11. Liu JJ, Bellamy G, Barnet B, Weng S. Bypass of local primary care in rural counties: effect of patient and community characteristics. Ann Fam Med. 2008; 6:124-30.

12. Coleman EA, Eilertsen TB, Kramer AM, Magid DJ, Beck A, Conner D. Reducing emergency visits in older adults with chronic illness. A randomized, controlled trial of group visits. Eff Clin Pract. 2001; 4:49-57.

13. Tudela P, Modol JM Urgencias hospitalarias. Med Clin (Barc). 2003; 120:711-6.

14. Baker DW, Stevens CD, Brook RH. Determinants of emergency department use by ambulatory patients at an urban public hospital. Ann Emerg Med. 1995; 25:311-6.

15. Consellería de Sanidade. Servizo Galego de Saúde. Memoria 2008 Sistema Público de Saúde de Galicia [Internet]. Santiago de Compostela: Xunta de Galicia. Consellería de Sanidade; 2009 [Acceso 11 de abril de 2011]. Disponible en: http://www.sergas.es/Publicaciones/ DetallePublicacion .aspx? IdPaxina $=40113 \&$ IDCatalo go $=1858$.

16. Oterino de la Fuente D, Baños Pino JF, Fernández Blanco $\mathrm{V}$, Rodríguez Álvarez A. Does better access to primary care reduce utilization of hospital accident and emergency departments? A time-series analysis. Eur J Public Health. 2007; 17:186-92.

17. Miró O, Salgado E, Tomas S, Espinosa G, Estrada C, Martí $C$, et al. Derivación sin visita desde los servicios de urgencias hospitalarios: cuantificación, riesgos y grado de satisfacción. Med Clin (Barc). 2006; 126:88-93.

18. Sempere T, Peiró S, Sendra P, Martínez C, López I. Validez del Protocolo de Adecuación de Urgencias Hospitalarias. Rev Esp Salud Publica. 1999; 73:465-79.

19. Sánchez M. ¿Urgencias inadecuadas u oferta insuficiente? Med Clin (Barc). 2004; 123:619-20. 